\title{
Darstellung der photometrischen und photographischen Größe als Funktion der Temperatur der Sterne.
}

\author{
Von Baron B. v. Harkanyi.
}

In meiner früheren A. N. 44 rg erschienenen Arbeit habe ich die visuelle photometrische Flächenhelligkeit $h$ des schwarzen Körpers als Funktion der absoluten Temperatur $T$ in der Form:

$$
\log h=A-\frac{B}{T}
$$

dargestellt. Aus dieser Formel folgt für die visuelle photometrische Größe der Sterne die. Gleichung:

$$
n=a+\frac{b}{T} \quad 5 \log \sin Q
$$

wo $b=2.5 \times B$ ist und $a$ durch die auf die Sonne bezüglichen Daten ${ }^{m} \odot, T_{\odot}$ und $\varrho_{\odot}$ bestimmt ist. Die im Draper-Kataloge enthaltenen photographischen Sterngrößen $m_{D}$ lassen sich durch die analoge Formel:

$$
m_{D}=\alpha+\frac{\beta}{T}-{ }_{5} \log \sin \varrho
$$

darstellen, wo:

$$
\beta=2.5 \log e \frac{c_{2}}{\lambda_{0}}, \quad \lambda_{0}=0.432 \mu
$$

und $c_{2}$ den Wert der Exponential-Konstanten der Wienschen Spektralgleichung darstellt. Diese Gleichung (3) gilt nur bei der vereinfachenden Annahme, daß der Stufenwert des DraperKataloges der Pogsonschen Zahl 0.4 entspricht; es kann dann $\alpha$ aus den Daten von Sternen bestimmt werden, für welche $m D, m$ und $T$ bekannt sind. Ist jedoch der Stufenwert von 0.4 verschieden und gleich $0.4: k$, so wird:

$$
m_{D}=k\left(\alpha+\frac{\beta}{T}-5 \log \sin \varrho\right) .
$$

Durch Elimination von $\varrho$ aus (2) und (5) folgt dann die Gleichung :

$$
{ }^{n} D-m=(k-\text { I }) m+k(\alpha-a)+k \frac{\beta-b}{T}
$$

und es müssen dann $\alpha$ und $k$ aus den Daten mehrerer Sterne von möglichst verschiedener Größe und Temperatur berechnet werden.

In dem folgenden habe ich versucht, diese Berechnung für Sterne auszufuhren, deren Temperaturen in der jüngst erschienenen Abhandlung von Scheiner und Wilsing ${ }^{1)}$ anf sehr exaktem spektralphotometrischen Wege bestimmt worden sind. bei der Rechnung sind, außer den Draper-Größen, die Daten der beiden genauesten Helligkeits-Kataloge: der Potsdamer Photometrischen Durchmusterung ${ }^{2}$ ) und der Revised Harvard Photometry ${ }^{3}$ ) benutzt worden, und zwar habe ich mich nicht nur auf die Konstanten $k$ und $\alpha$ beschränkt, sondern noch $\beta-b$ als dritte Unbekannte eingefïhrt, um die Gültigkeit der hier gemachten Annahmen prüfen zu können.

In der folgenden Tabelle I sind alle zur Rechnung notwendigen Daten nach steigender Temperatur der Sterne geordnet. Die erste Kolumne enthält die Nummer des Stemes nach dem Verzeichnisse von Scheiner und Wilsing, dann folgen die Benennung des Sternes, der Spektraltypus nach Vogel und Pickering, wo zur Abkürzung die Übergangstypen: II a-III a mit II a' und Ia 3 -II a mit Ia $3^{\prime}$ bezeichnet wurden, und die Farbenbezeichnung nach Potsdam. Die 5. Kolumne enthält die von Scheiner und Wilsing mit $T_{2}$ bezeichnete effektive Sterntemperatur dem Werte $\omega_{2}=$ I 4.6 entsprechend in tausend Graden angesetzt, welch letztere Einheit für $T$ ich bei allen folgenden Rechnungen beibehalten werde. Die 6. Kolumne enthält die photometrischen Größen $m_{P}$ nach Potsdam, die 7 . dieselben $m_{H}$ nach Harvard. Bei allen Sternen, die nach Harvard Annals 50, bei der Identifizierung mit dem Draper-Katalog als doppelt betrachtet worden sind, ist die in den Remarks zu dem ersteren angegebene kombinierte Sterngröße eingetragen worden. In zwei Fällen: bei $\iota$ Cancri und $\zeta$ Leonis findet sich auch im Potsdamer Katalog die zweite Komponente, die diesbezüglichen kleinen Korrektionen von -0.12 respektive - o.10 Größenklassen sind leider vor der Ausgleichung übersehen und erst nachträglich durchgeführt worden, was jedoch auf die Endresultate, wie ich mich iiberzeugt habe, einen verschwindend kleinen Einfluß ausübt. Die 8. Kolumne enthält die Differenz $m_{D}-m_{P}$, die ı. die Differenz $m_{D}-m_{H}$ für alle Sterne, für welche im Draper-Katalog verläßliche Größenangaben enthalten sind. Die drei Veränderlichen : $\alpha$ Orionis, $\alpha$ Herculis und $\eta$ Aquilae sind nicht aufgenommen worden und die wegen Unsicherheit der $m_{D}$ weniger verläßlichen; in Klammern gesetzten Differenzen bei den Ausgleichungen nicht benutzt worden.

\footnotetext{
) Publikationen des Astrophys. Observatoriums zu Potsdam Nr. 56 (Bd. I9, I. St.) und Astr. Nachr. Bd. 183 Nr. 4375.

Publikationen des Astrophys. Observatoriums zu Potsdam Bd. I7.

Annals of the Astron. Observatory of Harvard College Vol. 50.
} 
Tabelle I.

\begin{tabular}{|c|c|c|c|c|c|c|c|c|c|c|c|}
\hline $\mathrm{Nr}$. & Stern & Spektrum & Farbe & $T$ & $m_{P}$ & $m_{H}$ & $m_{D}-m_{P}$ & $\mathrm{~B}-\mathrm{R}$ & $\left|m_{D}-m_{H}\right|$ & $B-R$ & $s$ \\
\hline 20 & $\mu$ Geminorum & III a $\mathrm{Ma}$ & $\mathrm{R}$ & 2.8 & $3^{m} \circ 6^{\prime}$ & $3^{\mathrm{m} 2} \times 9$ & $+I^{m} \cdot 79$ & $-0^{m} \cdot 23$ & $+I^{m} \cdot 66$ & $-0^{\mathrm{m}} \cdot 30$ & o. 132 \\
\hline 64 & $\boldsymbol{x}$ Serper & III a $\mathrm{K}_{5}$ & $\mathrm{G}$ & 2.8 & 4.10 & 4.28 & +1.76 & -0.03 & $+1.5^{8}$ & -0.10 & 0.100 \\
\hline 5 & $\beta$ Andromed. & $\mathrm{IIa}^{\prime} \mathrm{Ma}$ & $R G-$ & 2.9 & $2 \cdot 3^{2}$ & 2.37 & +2.25 & +0.16 & +2.20 & +0.30 & 0.2 i 3 \\
\hline 33 & 40 Lyncis & $\mathrm{IIa}^{\prime} \mathrm{K}_{5}$ & $\mathrm{G}+$ & 3.0 & $3 \cdot 3^{8}$ & $3.3^{\circ}$ & +1.60 & -0.17 & $+\mathrm{r} .68$ & -O.IO & 0.230 \\
\hline 44 & $\nu$ & III a $\mathrm{Ma}$ & $\mathrm{G}$ & 3.0 & 4.20 & 4.20 & +1.25 & -0.34 & +1.25 & -0.30 & .84 \\
\hline 47 & $\delta$ Virgini & $\operatorname{IIIa~} \mathrm{Ma}$ & $\mathrm{G}-$ & 3.0 & 3.64 & 3.66 & +1.72 & +0.01 & +1.70 & $+0.0 \mathrm{I}$ & 485 \\
\hline $5^{I}$ & $v$ Bootis & IIIa $K_{5}$ & $\mathrm{G}-$ & 3.1 & 4.07 & 4.28 & +1.57 & +0.02 & $+1 \cdot 3^{6}$ & -0.10 & I $2 \mathrm{I}$ \\
\hline 90 & $\delta$ Sag & IlIa Map & $\mathrm{G}-$ & 3.1 & 3.86 & 3.78 & $+\mathbf{I}$ & -0.01 & +1.66 & +0.07 & .029 \\
\hline 28 & $\beta$ Can & $\mathrm{IIa}^{\prime} \mathrm{K}_{2}$ & $\mathrm{G}-$ & 3.2 & 3.74 & 3.76 & $+1 \cdot 3^{2}$ & -0.23 & $+r \cdot 30$ & -0.23 & 0.072 \\
\hline 89 & $\gamma \mathrm{Aq}$ & $\mathrm{IIa}^{\prime} \mathrm{K}_{2}$ & $\mathrm{G}-$ & $3 \cdot 3$ & 3.08 & 2.80 & $+1.5^{8}$ & -0.04 & +1.86 & +0.14 & .012 \\
\hline 94 & $\gamma \mathrm{Sag}$ & $\mathrm{IIa}^{\prime} \mathrm{K}_{5}$ & $\mathrm{G}-$ & $3 \cdot 3$ & 3.83 & $3.7 \mathrm{I}$ & $+1.5^{\circ}$ & +0.04 & +1.62 & +0.14 & .068 \\
\hline 102 & $\varepsilon$ Pegasi & $\mathrm{IIa}^{\prime} \mathrm{K}$ & G -- & $3 \cdot 3$ & 2.76 & 2.54 & +1.65 & -0.04 & +1.87 & +0.08 & $.03 \mathrm{I}$ \\
\hline 3 & d Andron & $\mathrm{IIa}^{\prime} \mathrm{K}$ & $\mathrm{G}$ & $3 \cdot 5$ & 3.48 & 3.49 & $+1 \cdot 31$ & -0.10 & $+\mathrm{r} \cdot 3^{\circ}$ & $-0 . \times 3$ & 169 \\
\hline 53 & $\alpha$ Bootis & $\mathrm{IIa}^{\prime} \mathrm{K}$ & $W G$ & $3 \cdot 5$ & 0.24 & 0.24 & - & - & - & - & 2.277 \\
\hline 107 & $\lambda$ Peg & $\mathrm{IIa}^{\prime} \mathrm{K}$ & WG & $3 \cdot 5$ & 4.19 & 4. I 4 & + I. $\times 5$ & -0.10 & +1.20 & -0.06 & 62 \\
\hline 75 & $\pi \mathrm{I}$ & $\mathrm{II} \mathrm{a}^{\prime} \mathrm{K}_{\mathbf{2}}$ & $\mathrm{G}-$ & 3.6 & $3 \cdot 32$ & $3 \cdot 3^{6}$ & $+\mathrm{I}$ & +0.28 & +1.62 & $+0.2 \mathrm{I}$ & $.03 x$ \\
\hline 6 & $\eta \mathrm{F}$ & $\mathrm{IIa}^{\prime} \mathrm{G}_{5}$ & WG & $3 \cdot 7$ & 4.07 & 3.72 & +0.95 & $-0.2 x$ & +1.30 & +0.03 & .017 \\
\hline 9 & $\alpha A$ & $\mathrm{II} \mathrm{a}^{\prime} \mathrm{K}$ & WG & 3.7 & 2.19 & 2.23 & +1.94 & +0.37 & +1.90 & +0.25 & .240 \\
\hline 43 & $\nu$ Ursa & $\mathrm{IIa}^{\prime} \mathrm{K}$ & $\mathrm{G}+$ & $3 \cdot 7$ & 3.62 & 3 & +1 & +0.27 & $+\mathrm{r} .44$ & +0.17 & 0.046 \\
\hline 62 & $\alpha$ Ser & $\mathrm{IIa}^{\prime} \mathrm{K}$ & $\mathrm{G}-$ & $3 \cdot 7$ & 2.88 & 2.75 & $+\mathrm{I}$ & -0.07 & +1.48 & -0.04 & .144 \\
\hline $3^{6}$ & $\mu \mathrm{I}$ & $\mathrm{IIa}^{\prime} \mathrm{K}$ & & 3.8 & 4.08 & 4.10 & +1 & +0.18 & $+r .27$ & +0.15 & 0.233 \\
\hline$\times 5$ & $\delta^{\prime}$ & $\mathrm{IIa}^{\prime} \mathrm{K}$ & & $3 \cdot 9$ & 4. 16 & 3.93 & $+c$ & -0.20 & + & -0.05 & 一 \\
\hline 77 & $\beta c$ & $\mathrm{IIa}^{\prime} \mathrm{K}$ & $\mathrm{G}-$ & 4.0 & 3.06 & 2.94 & $+I . I 3$ & -0.10 & +1.25 & -0.09 & 0.165 \\
\hline 80 & $* 1$ & $\mathrm{IIa}^{\prime} \mathrm{K}$ & WG & 4.0 & 3.95 & 3.99 & $+\mathrm{I} .59$ & +0.55 & $+\mathrm{r} .55$ & +0.48 & .014 \\
\hline 10 & o Tauri & $\mathrm{IIa}^{\prime} \mathrm{G}_{5}$ & $\mathrm{GW}$ & $4 . I$ & 3.86 & 3.80 & +0.92 & - o.Io & +0.98 & -0.10 & .095 \\
\hline 29 & $\delta$ Cancri & $\mathrm{IIa}^{\prime} \mathrm{K}$ & WG & $4 \cdot I$ & 4.04 & 4.17 & +1.22 & +0.24 & +1.09 & $+0.1 x$ & .237 \\
\hline $3 \circ$ & $\iota$ Cancri & $\mathrm{II}^{\prime} \mathrm{G}_{5}$ & G & $4 . I$ & 4.06 & 4.09 & +0.79 & -0.18 & +0.76 & -0.24 & .042 \\
\hline 35 & $\varepsilon$ & $I I a^{\prime} G p$ & & 4. I & 3.22 & 3.12 & +1 & -0.04 & + & -0.23 & .042 \\
\hline $5^{6}$ & $\varepsilon$ Boc & II $a^{\prime} K$ & WG & 4. I & 2.66 & 2.59 & $(+1.22)$ & - & $(+1.29)$ & - & .041 \\
\hline 85 & 109 & $\mathrm{II} \mathrm{a}^{\prime} \mathrm{K}$ & $W G+$ & $4 \cdot \mathrm{T}$ & 4.06 & 3.92 & +1.08 & $+0.1 \mathrm{I}$ & +1.22 & +0.17 & 32 \\
\hline 98 & $\varepsilon$ Су & II $a^{\prime} K$ & $\mathrm{G}$ & 4. I & 2.72 & 2.64 & $+\mathrm{I} \cdot \mathrm{r} 3$ & - O.I 3 & $+1.2 I$ & -0.17 & 0.494 \\
\hline 18 & $\varphi^{2} \mathrm{O}$ & II $\mathrm{a}^{\prime} \mathrm{K}$ & $W G$ & 4.2 & 4. & 4. & +1 & +0.18 & +0.89 & +0.01 & 0.318 \\
\hline $3^{2}$ & & $\mathrm{IIa}^{\prime} \mathrm{K}$ & WC & 4.2 & $3 \cdot 34$ & & + & 0.00 & $+\mathrm{I} . \mathrm{I} 2$ & -0.05 & $.09 \mathrm{I}$ \\
\hline 58 & $\delta \mathrm{I}$ & $\operatorname{II} \mathbf{a}^{\prime} \mathrm{K}$ & $w$ & 4.2 & 3 & 3.54 & +1 & +0.10 & $+\mathrm{I} .18$ & +0.07 & .153 \\
\hline 71 & $x$ & II $a^{\prime} \mathrm{K}$ & WG & 4.2 & $3 \cdot 3^{6}$ & $3 \cdot 4^{2}$ & +1.20 & +o.1 2 & $+\mathrm{I} . \mathrm{I} 4$ & 0.00 & .295 \\
\hline 106 & $\eta \mathrm{I}$ & II $a^{\prime} G$ & $\mathrm{G}$ & 4.2 & 3.22 & 3.10 & +0.94 & -0.17 & +1.06 & -0.16 & .038 \\
\hline 24 & inorum & IIIa K & $\mathrm{G}-$ & $4 \cdot 3$ & 3.96 & 3.89 & +0.92 & $+0.0 \mathrm{I}$ & +0.99 & $+0.0 \mathrm{r}$ & 134 \\
\hline 49 & $\varepsilon V$ & $\mathrm{IIa}^{\prime} \mathrm{K}$ & WG & $4 \cdot 3$ & 3. & 2.95 & $+x .6 r$ & $+0.5 \mathrm{I}$ & +1.77 & +0.54 & 0.264 \\
\hline I 4 & $\gamma \mathrm{T}$ & $I I a^{\prime} G$ & W & 4 & 3. & & +0.87 & 0.00 & + & +0.04 & - \\
\hline 26 & $x($ & II a' $\mathrm{C}_{5}$ & $\mathrm{G}$ & 4.4 & 3.75 & 3.68 & +0.87 & -0.05 & + & -0.06 & 0.066 \\
\hline 27 & $\beta \mathrm{G}$ & $\mathrm{IIa}^{\prime} \mathrm{K}$ & WG & 4.4 & I. $5 \mathrm{I}$ & I. 21 & $(+1.90)$ & - & $(+2.20)$ & - & 617 \\
\hline 93 & $\beta$ & $\mathrm{IIa}^{\prime} \mathrm{K}$ & WG & 4.4 & 3.83 & 3.90 & $+\mathrm{r} .0 \mathrm{I}$ & $+0.1 \mathrm{I}$ & +0.93 & -0.01 & $.48 \mathrm{I}$ \\
\hline rog & $\gamma \mathrm{F}$ & $\mathrm{II} \mathrm{a}^{\prime} \mathrm{K}$ & WG & 4.4 & 4.08 & 3.85 & +0.95 & $+0.1 I$ & +1.18 & +0.23 & .750 \\
\hline 68 & $\beta \mathrm{I}$ & $\mathrm{IIa}^{\prime} \mathrm{K}$ & WG- & 4.6 & $3.0 \mathrm{r}$ & $2.8 \mathrm{I}$ & +1.16 & +0.16 & $+1.3^{6}$ & +0.20 & O. I I I \\
\hline 99 & $\zeta 0$ & $\mathrm{IIa}^{\prime} \mathrm{K}$ & $G$ & $4 \cdot 7$ & & 3.40 & +0.97 & +0.09 & +1.02 & +0.04 & 0.067 \\
\hline 83 & 70 & $\mathrm{IIa}^{\prime} \mathrm{K}$ & WG & 4.8 & 4.12 & 4.07 & +0.95 & +0.25 & +1.00 & +0.23 & I. I 34 \\
\hline $3 I$ & & $\mathrm{IIa} F 8$ & WG & 4.9 & $3 \cdot 57$ & $3 \cdot 4^{8}$ & +0.74 & -0.05 & +0.83 & -0.07 & о. I 84 \\
\hline 8 I & $\xi$ & $\mathrm{IIa}^{\prime} \mathrm{K}$ & WG & 4.9 & 3.88 & 3.82 & +0.91 & +0.19 & +0.97 & +0.16 & - \\
\hline $5^{2}$ & $\eta \mathrm{I}$ & II a $G$ & & 5.0 & 3. & 2.80 & $+0.7 \mathrm{I}$ & -0.15 & +0.99 & -0.06 & $0.36 I$ \\
\hline 70 & & II $a^{\prime} \mathrm{K}$ & & 5.1 & $3.7^{6}$ & $3.6 \mathrm{I}$ & +0.89 & +0.20 & +1.04 & +0.22 & 0.098 \\
\hline 78 & & $\mathrm{II} \mathrm{G}_{5}$ & & 5.2 & 3.62 & 3.48 & +0.77 & +0.08 & $+0.9 \mathrm{I}$ & +0.08 & 0.806 \\
\hline 103 & & II a $\mathrm{F}_{5}$ & $\mathrm{GW}+$ & 5.2 & 4.00 & 3.96 & +0.44 & -0.17 & +0.48 & -0.22 & 0.303 \\
\hline 34 & onis & $\mathrm{Ia}_{3}^{\prime} \mathrm{F} 5 \mathrm{p}$ & GW & $5 \cdot 3$ & 3.88 & 3.76 & $+0.3^{8}$ & -0.23 & $+0.5^{0}$ & -0.23 & $0.13^{6}$ \\
\hline . & $\alpha$ Trianguli & Ia $3^{\prime} F_{5}$ & WG- & $5 \cdot 5$ & 3.64 & $3 \cdot 5^{8}$ & +0.67 & +0.06 & +0.73 & 0.00 & 0.230 \\
\hline 16 & 3 Orionis & $\mathrm{Ia}_{3}^{\prime} \mathrm{F} 8$ & GW & $5 \cdot 5$ & 3.50 & $3.3 \mathrm{I}$ & 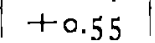 & -0.09 & 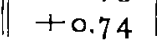 & 7 & 0.465 \\
\hline
\end{tabular}




\begin{tabular}{|c|c|c|c|c|c|c|c|c|c|c|c|c|}
\hline Nr. & Stern & pek & trum & arbe & $T$ & $m_{P}$ & $m_{H}$ & $m_{D}-m_{P}$ & $B-R$ & $m_{D}-m_{H}$ & $B-R$ & $s$ \\
\hline 60 & $\zeta$ Herc & a & $\mathrm{G}$ & $V G-$ & 5 & $3^{\mathrm{m}} \cdot \mathrm{r}$ & $3^{m} \cdot 00$ & $+0^{\mathrm{m}} \cdot 77$ & $+0^{m} .06$ & $+0^{m} \cdot 93$ & $+0^{m} \cdot 04$ & o." 596 \\
\hline 23 & d Geminorum & I a 3 & $\mathrm{~F}$ & WG & 5 & & & +0.44 & -0.14 & +0.62 & -O.II & .017 \\
\hline 22 & $\xi$ Geminorum & I a $3^{\prime}$ & $\mathrm{F}_{5}$ & GW- & $5 \cdot 7$ & 3.63 & 3.40 & .35 & $-0.2 \mathrm{I}$ & $+0.5^{8}$ & -0.16 & 0.231 \\
\hline 95 & r Cygni & II a & $\mathrm{F} 8 \mathrm{p}$ & WG & $5 \cdot 7$ & 2.48 & $2 \cdot 3^{2}$ & +0.68 & -0.13 & +0.84 & -0.18 & 0.019 \\
\hline ror & $\alpha$ Equulei & I a $3^{\prime}$ & A $8 p$ & GW - & $5 \cdot 7$ & 4.08 & 4.14 & +0.56 & +0.10 & $+0.5^{\circ}$ & -0.04 & 0.094 \\
\hline 66 & $\gamma$ Serpentis & I a $3^{\prime}$ & F 8 & GW- & 5.8 & 4.07 & 3.86 & +0.47 & +0.03 & & +0.08 & I. 290 \\
\hline 54 & $\gamma$ Bootis & I a 2 & $\mathbf{F}$ & $W+$ & 6.0 & $3 \cdot 34$ & 3.00 & $+0.1 \mathrm{I}$ & -0.46 & +0.45 & -0.34 & 0.180 \\
\hline I 2 & o Persei & Ib? & $\mathrm{B}_{\mathrm{I}}$ & $\mathrm{GW}+$ & 6.1 & & & & +o.II & & & - \\
\hline 46 & $\beta$ Virginis & II a & F 8 & GW - & 6.1 & & & & $+0.1 I$ & & +0.04 & 0.790 \\
\hline 67 & $\gamma \mathrm{Her}$ & I $a 3$ & $\mathrm{~F}$ & GW -- & 6.2 & .3 .97 & 3.79 & & -0.03 & 34 & 0.00 & 0.064 \\
\hline 96 & $\beta \mathrm{Dc}$ & $\mathrm{Ia}_{3}$ & $\mathrm{~F}_{5}$ & $\mathrm{GW}^{T}$ & 6.3 & 4.02 & $3.7^{2}$ & +0.36 & 0.00 & & +o. I I & 0.107 \\
\hline 88 & $\delta \mathrm{Aq}$ & I a $3^{\prime}$ & $\mathrm{F}$ & $\mathrm{GW}$ & 6.4 & 3.74 & $3 \cdot 44$ & +0.29 & $-0.1 \mathrm{I}$ & +0.59 & $-0.0 \mathrm{I}$ & 0.264 \\
\hline 4 & $\mu$ And & I a 3 & $\mathrm{~A}_{2}$ & $W+$ & 6.5 & 4.03 & 3.94 & +0.40 & +0.08 & & +0.03 & 0.188 \\
\hline 39 & $\zeta$ Leonis & I a 3 & $\mathrm{~F}$ & WG & 6.6 & 3 & & & +0.07 & & $+0.0 \mathrm{I}$ & 0.020 \\
\hline $4 \mathrm{I}$ & $d$ Leonis & I a $3^{\prime}$ & $A_{2}$ & GW & 6.9 & & & & -0.22 & & -0.14 & 0.206 \\
\hline 13 & $17 \mathrm{Ta}$ & $\mathrm{Ib}$ & $\mathrm{B}_{5}$ & V & 7.1 & & & & $-0.2 I$ & & -- о. І 9 & - \\
\hline 76 & $\alpha \mathrm{Opl}$ & I 2 & $A_{5}$ & GW- & 7.1 & 2.54 & 2. I 4 & +0.34 & $-0.2 I$ & 74 & $-0.1 \mathbf{I}$ & 0.254 \\
\hline 91 & $\alpha \mathrm{Aq}$ & I a $3^{\prime}$ & A 5 & GW- & $7 \cdot I$ & 1.12 & 0.89 & $(+$ & - & 82) & - & $0.65^{6}$ \\
\hline $6_{3}$ & $\beta$ Ser & I a 2 & $\mathrm{~A}_{2}$ & GW- & $7 \cdot 3$ & 3.84 & $3 \cdot 74$ & 0.00 & -0.24 & $+c$ & -0.30 & 0.079 \\
\hline 100 & $\tau$ Cygni & I a 3 & $\mathrm{~F}$ & GW & $7 \cdot 3$ & & & & +0.18 & & +0.11 & 0.477 \\
\hline 59 & $\beta \mathrm{Co}$ & I a $3^{\prime}$ & $F p$ & W & 7.6 & & & & +0.07 & & 0.06 & 0.172 \\
\hline 74 & d' Hercu & I a 2 & A & $w+$ & $7 \cdot 7$ & & 3.10 & +0.24 & -0.03 & & -0.03 & . 168 \\
\hline 48 & I2 $\mathrm{Can}$ & I a $\mathrm{I}$ & $A p$ & $\mathrm{GW}+$ & 7.8 & 3.10 & 2.80 & $(+0.04)$ & - & 34) & - & 0.247 \\
\hline 57 & 109 & I a 2 & A & GW & 7.8 & 4.00 & $3.7^{6}$ & +0.07 & -0.07 & $.3 \mathrm{I}$ & -0.03 & 0.135 \\
\hline I & ce And & I a 2 & A & W & 8.0 & 2.42 & 2.15 & $37)$ & - & $(+0.64)$ & - & 0.209 \\
\hline 65 & $\varepsilon$ Serpc & la 2 & A & Gr & 8.0 & 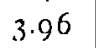 & & +0.08 & -0.05 & +0.29 & -0.03 & $0.13 I$ \\
\hline 45 & $\beta \mathrm{Le}$ & I a 2 & $\mathrm{~A}_{2}$ & & 8.1 & 2.02 & & - & - & - & - & \\
\hline 25 & $\beta \mathrm{Ca}$ & I a I & B 8 & & 8.4 & 3.20 & 3.09 & +0.15 & -0.10 & 0.26 & -0.20 & 0.062 \\
\hline 42 & $\vartheta \mathrm{Le}$ & I a 2 & A & $I-$ & 8.4 & 3.58 & $3.4 \mathrm{I}$ & +0.03 & -0.13 & & -0.17 & 0.095 \\
\hline 86 & $\gamma \mathrm{L}, \mathrm{y}$ & I a 2 & $A$ & $V+$ & 8.6 & 3.55 & $3 \cdot 3^{\circ}$ & -0.02 & - O.I 7 & 23 & -0.15 & 0.008 \\
\hline 79 & $\gamma \mathrm{O}_{\beta}$ & I a 2 & $\mathrm{~A}$ & GW-- & 8.7 & 4. & & - & -0.29 & .09 & -0.17 & $0.07 \mathrm{I}$ \\
\hline 8 & a Pis & I a 2 & $\mathrm{~A} 2$ & GW - & S.8 & & & - & -0.04 & & --0.05 & 0.046 \\
\hline 72 & $\varepsilon \mathrm{He}$ & I a 2 & A & & 8.8 & & 3. & -0.28 & -0.28 & .04 & -0.25 & $0.05 \mathrm{I}$ \\
\hline 2 & $\gamma \mathrm{Po}$ & Ib & $\mathrm{B}_{2}$ & - & 8.9 & 3.28 & 2.87 & 18 & 0.00 & +0.59 & +0.12 & 0.017 \\
\hline 104 & $\vartheta \mathrm{Pe}$ & I a 2 & A & GW-- & 8.9 & 4.08 & 3.70 & +0.14 & $+0 . I_{4}$ & $.5^{2}$ & +0.27 & 0.285 \\
\hline $6 \mathrm{r}$ & $\gamma$ Cor & I a 2 & A & $W+$ & $9 . \mathrm{I}$ & 3.98 & 3. & +0.15 & + o. I 4 & +0.20 & +0.02 & 0.099 \\
\hline 37 & $\eta$ Lec & $\mathrm{Ib}$ & Ap & GW- & 9. & 382 & & +0.08 & +0.05 & & +0.06 & .040 \\
\hline 40 & e I & I & B & & 9 & & & & +0.01 & & .05 & 07 \\
\hline TO5 & & I & B & & 9. & 3.1 & & +0.05 & 0.00 & +0.18 & -0.07 & 0.088 \\
\hline 38 & \& Le & & B 8 & GW & 9.4 & r. 7 & & - & - & - & - & 0.245 \\
\hline 84 & o $\mathrm{He}$ & I a 2 & $A$ & W & 9.5 & 4.06 & 3.83 & 0.00 & -0.04 & +0.23 & -0.06 & 0.007 \\
\hline 60 & $\ll \mathrm{C}$ & $\mathrm{Ia}_{2}$ & A & W & 9.6 & $2.5^{8}$ & $2.3 I$ & $(+0.87)$ & - & $(+\operatorname{II} 4)$ & - & 0.162 \\
\hline I I & $\xi$ Tauri & I a 2 & B 8 & GW -- & 9.8 & 3.94 & $3 \cdot 7$ & +0.42 & +0.46 & $+0.6 \mathrm{I}$ & +0.43 & $0.09 \mathrm{r}$ \\
\hline $5^{\circ}$ & & $\mathrm{Ia} 2$ & $\mathrm{~A} 2$ & $\mathrm{GW}-$ & 9.8 & 6 & & +0.45 & $+0.4 \mathrm{r}$ & +0.57 & +0.31 & 0.292 \\
\hline$s_{7}$ & $\zeta \mathrm{A}$ & I $\mathrm{a}_{2}$ & $A$ & GW - & 9.8 & & & & +0.13 & 52 & +0.15 & 0.097 \\
\hline $8 z$ & 670 & $\mathrm{Ib}$ & $\mathrm{B}_{5}$ & GW - & 10.0 & $4 \cdot 18$ & 3.9 & +0.03 & +0.14 & +0.29 & +0.17 & 0.046 \\
\hline $2 \mathrm{I}$ & $\gamma \mathrm{Ge}$ & I $\mathrm{Ia}_{2}$ & A & & I 0.3 & $2 \cdot 34$ & & $(+0.60)$ & - & $(+\mathrm{I} .0 \mathrm{I})$ & - & 0.066 \\
\hline 55 & $\zeta$ Bootis & I a 2 & $\mathrm{~A}_{2}$ & GW- & 10.4 & 4.04 & 3.86 & -0.02 & +0.08 & +0.16 & +0.05 & 0.049 \\
\hline 97 & \& Delphini & I a I & B 8 & GW- & 10.7 & 4.14 & 3.86 & -0.35 & -0.20 & -0.07 & -0.16 & 0.064 \\
\hline 08 & $\alpha$ Pegasi & I a I & A & GW - & I I.5 & 3.20 & 2.57 & $(+0.01)$ & - & $(+0.64)$ & - & 0.067 \\
\hline I 7 & 2 Orionis & $\mathrm{Ib}$ & $\mathrm{Oe}_{5}$ & GW- & I 2.8 & 3.70 & 3.49 & -0.24 & -0.08 & -0.03 & -0.12 & $0.03 \mathrm{I}$ \\
\hline
\end{tabular}

Zur Durchführung der Rechnung sind für die dazu angesetzt und mit gleichem Gewichte zur Ableitung der Werte verwendbaren 95 Sterne die Fehlergleichungen für die $m_{P^{-}}$ Größen in der Form:

$$
m_{D}-m_{P}=x+\frac{y^{y}}{T}+z m_{P}
$$
der drei Unbekannten nach der Methode der kleinsten Quadrate verwendet worden. Das Resultat der Rechnung nebst den mittleren Fehlern ist folgendes: 


$$
\begin{aligned}
& x=+0.0634 \pm 0.178 \\
& y=+7.316 \pm 0.268 \\
& z=-0.2155 \pm 0.043^{\circ}
\end{aligned}
$$

und der mittlere Fehler einer Gleichung $\mu= \pm 0^{\mathrm{m}}$. 86 . Die Differenzen im Sinne Beobachtung - Rechnung stehen in der 9. Kolumne angesetzt. Um den Einfluß des $z$-Gliedes $z u$ untersuchen, habe ich versucht, $z$ ganz zu unterdrücken und die Fehlergleichungen in der Form:

$$
m_{D}-m_{P}=x+\frac{y}{T}
$$

auszugleichen, es wird dann:

$$
\begin{gathered}
x=-0.783 \pm 0.062 \\
y=+7.608 \pm 0.292 \\
\mu= \pm 0^{\mathrm{m}} 208 .
\end{gathered}
$$

Die Darstellung wird nicht wesentlich verschlechtert, wie es die nur unbedeutende Vergrößerung von $\mu$ anzeigt, $y$ ändert sich auch nicht viel, auffallend ist aber die beträchtliche Verminderung des mittleren Fehlers von $x$.

Auf vollkommen gleiche Art sind die $m_{H}$ entsprechenden 95 Fehlergleichungen von der Form:

$$
m_{D}-m_{H}=x+\frac{y}{T}+z m_{H}
$$

behandelt worden und geben die Resultate:

$$
\begin{gathered}
x=+0.498 \pm 0^{m} \mathrm{r}^{\mathrm{I}} \\
y=+6.44 \mathrm{I} \pm 0.243 \\
z=-0.26 \mathrm{I} \pm 0.0365 \\
\mu= \pm 0 . \mathrm{r} 73 .
\end{gathered}
$$

Die Differenzen im Sinne Beobachtung - Rechnung sind in der I I. Kolumne angeführt.

Nach Weglassung des $z$-Gliedes wird in diesem Falle

$$
\begin{gathered}
x=-0^{\mathrm{m}} .439 \pm 0^{\mathrm{ml}} .063 \\
y=+6.5 \mathrm{I0} \pm 0.297 \\
\mu= \pm 0^{\mathrm{m} \cdot 2 \mathrm{I} 2}
\end{gathered}
$$

die zu ähnlichen Bemerkungen Veranlassung geben.

Die Darstellung der Beobachtungen durch die so abgeleiteten Formeln (7). und (I I) erscheint im allgemeinen durchaus genügend, und die Abweichungen scheinen keinerlei systematische Fehler anzuzeigen. Auffallend ist der Parallelismus der Differenzen für $m_{p}$ und $m_{H}$, woraus ich schließe, daß alle größeren Abweichungen durch die $m_{D}$ verursacht worden sind. $O b$ diese Erscheinung von größeren zufälligen Fehlern der Draper-Größen herriihrt, oder etwa besonderen Eigentümlichkeiten der betreffenden Sterne zuzuschreiben ist, läßt sich auf Grund des vorliegenden kleinen Materials nicht entscheiden.

Zur Beurteilung der mittleren Fehler $\mu$ möchte ich nur erwähnen, daß in $\boldsymbol{\mu}$ außer den mittleren Fehlern der beiden Katalogwerte noch der mittlere Fehler von $\frac{\mathrm{I}}{T}$ eintritt; da nach Scheiner und Wilsing ${ }^{1}$ ) der mittlere Fehler von $T$, in der hier benutzten Einheit ausgedrückt, $\pm 0.00954 T^{2}$ ist, so folgt daraus der Teil des mittleren Fehlers von $\frac{y}{T}$, der nur von der Unsicherheit von $T$ herrührt, $\pm 0^{\mathrm{m}} .0698$ für (7), respektive \pm 0.0616 für ( $1 \mathrm{r}$ ); die Quadrate dieser Größen müßten noch zur Quadratsumme der beiden mittleren Katalogfehler hinzuaddiert werden, um $\mu^{2}$ zu erhalten. Kapteyn ${ }^{2}$ ) findet $\mu= \pm 0.2$ I 9 bei seiner Ausgleichung der Differenzen $m_{D}-m_{H}$

Die Bestimmung der $z$ ist wegen des zienlich mäßigen Intervalles von etwa zwei Größenklassen, in welchem die $m_{P}$ respektive $m_{H}$ variieren, nicht sehr günstig, doch sind die Resultate nach dem Ausweise der mittleren Fehler ziemlich sicher. Der hier gefundene Wert von $z$ in (1 2 ) liegt etwa in der Mitte der von Kapteyn für die einzelnen Spektraltypen getrennt berechneten Werte ${ }^{3}$ ), die für die Cannon-Sterne zwischen -0.196 (für $B_{3}$ bis $B_{9}$ ) und $-0.33^{6}$ (für $K$ ) liegen, für die Maury-Sterne zwischen - $0 . \mathrm{I}_{43}$ (für $\mathrm{B}_{3}$ bis A 2) und -0.364 (für $\mathrm{K}$ bis $\mathrm{Ma}$ ). Derartige Schwankungen dieser Größe scheinen bei dem vorliegenden Material nicht sehr wahrscheinlich und durch den Gang der Fehler nicht angedeutet, eine direkte Prüfung der Konstanz von $z$ bei den verschiedenen Spektraltypen an diesem zu geringen Material kaum durchführbar.

Zur besseren Übersicht sind in Tabelle II die mit den Konstanten ( 10 ) respektive (i 2 ) berechneten Differenzen $m_{D}-m_{P}$ und $m_{D}-m_{H}$ für eine Reihe von Temperaturen zusammengestellt.

Tabelle II.

\begin{tabular}{c|c|c||c|c|c}
\hline$T$ & $m_{D}-m_{P}$ & $m_{D}-m_{H}$ & $T$ & $m_{D}-m_{P}$ & $m_{D}-m_{H}$ \\
\hline 2.0 & $+2^{\mathrm{m}} .97$ & $+2^{\mathrm{m}} .80$ & 8.0 & +0.22 & +0.39 \\
3.0 & $+\mathrm{I} .74$ & $+\mathrm{I} .73$ & 9.0 & +0.12 & +0.30 \\
4.0 & +1.14 & +1.19 & 10.0 & +0.04 & +0.23 \\
5.0 & +0.77 & +0.87 & $1 \times .0$ & -0.02 & +0.17 \\
6.0 & +0.53 & +0.66 & $\mathrm{I} 2.0$ & -0.08 & +0.12 \\
7.0 & +0.36 & +0.50 & $\infty$ & -0.69 & -0.42
\end{tabular}

Die Daten der Tabelle beziehen sich auf die Größe 3.5 - nahe dem Mittel der benutzten visuellen Sterngrößen und können durch Hinzufügung der Korrektionsglieder: $+0.216\left(3^{m} \cdot 5^{0}-m_{P}\right)$ respektive $+0.26 \mathrm{I}\left(3^{\mathrm{m}} \cdot 5^{\circ--} m_{H}\right)$ auf beliebige Größen umgerechnet werden.

Zur Berechnung von $T$ können, wie in A. N. 4419 Gl. ( 18 ), die Gleichungen:

$$
\left.\begin{array}{l}
T=7.316 \frac{\mathrm{I}}{m_{D}-m_{P}+0.2 \mathrm{r} 6 m_{P}-0.063} \\
T=6.44 \mathrm{I} \frac{\mathrm{r}}{m_{D}-m_{H}+0.26 \mathrm{r} m_{H}-0^{\mathrm{m}} 49_{8}}
\end{array}\right\}(\text { I } 4)
$$

dienen, deren Gültigkeitsbereich dadurch begrenzt ist, daß die Nenner nicht negative Werte annehmen dürfen. Die in meiner früheren Mitteilung gegebene graphische Darstellung der $m_{D}$ und $m_{P}$ oder $m_{D}$ und $m_{H}$ läßt sich nach dem bisherigen leicht ausführen. Die Geraden $T=$ konst. wurden dann durch die Gleichungen:

1) Publikationen des Astrophys. Observatoriums zu Potsdam Nr. 56 p. 63

$\Rightarrow$ On the absorption of light in space. Contrib. from the Mt. Wilson Solar Obs. No. 42 und Astroph. J. 30.285 
$m_{D}=0.784 m_{P}+$ konst.; $\quad m_{D}=0.739 m_{H}+$ konst.

dargestelit und ihre Neigungswinkel gegen die Abszissenachse von $45^{\circ}$ verschieden und zwar gleich $38^{\circ}$. r respektive $36^{\circ} .5$. Der Koeffizient von $m_{H}$ unterscheidet sich wicht wesentlich von dem aus dem Mittel von Hertzsprungs Zahlen berechneten Werte (A. N. 4419 p. 45).

$\mathrm{Un}$ aus den Gleichungen von der Form (7) und ( $\mathrm{x}$ I) die Formeln von der Gestalt (2) und (5) abzuleiten, können die bisherigen Ergebnisse verschiedenartig kombiniert werden. Wenn die Konstanten in ( 7 ) mit dem Index $I$, die in ( $I x$ ) mit dem Index 2 bezeichnet werden, so folgt mit Rücksicht auf (6) leicht:

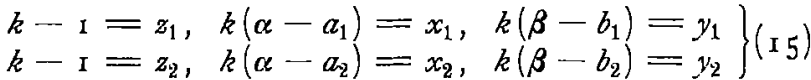

wo $a$ und $b$ analog durch Indexe unterschieden sind. Es folgt somit, daß $k$ nicht widerspruchsfrei aus beiden Ausgleichungen berechnet werden kann. Da jedoch die Differenz $z_{1}-z_{2}$ nach Ausweis der mittleren Fehler kaum zu verbürgen ist und ein den $m_{H}$ proportionales Glied in $m_{P}-m_{H}$ einführen würde, dessen Koeffizient -0.062 wäre und ein so großer Wert nach den eingehenden Untersuchungen von Mïller und Kempf ${ }^{1}$ ) sehr unwahrscheinlich ist, habe ich es vorgezogen, diese Differenz zu vernachlässigen, und $k$-- I gleich dem Mittel von $z_{1}$ und $z_{2}$ zu nehmen, welches gleich -0.2383 ist. $\mathrm{Zu}$ den Gleichungen ( ${ }_{5}$ ) kommen noch die aus (2) für $m_{P}$ und $m_{H}$ folgenden Relationen, die durch Einsetzung der Werte $m_{\odot}, T_{\odot}$ und $\varrho_{\odot}$ entstehen. Da die bisherigen Gleichungen jedoch nicht hinreichen, um die sechs Konstanten $a_{1}, b_{1}, a_{2}, b_{2}, \alpha, \beta$ zu berechnen, habe ich es vorgezogen, für $\beta$ den mit $c_{2}=\mathrm{x}_{4} .6$ aus $(4)$ folgenden Wert $\beta=3^{6.963}$ anzunehmen, da $c_{2}$ ohnehin eine für diese Untersuchungen unentbehrliche physikalische Konstante bildet, weil ja die $T_{2}$-Werte auch auf dieser Konstanten beruhen, die durch die Arbeit von Scheiner und Wilsing durchaus genügend verifiziert ist. Durch diese Festsetzungen wird das Problem überbestimmt: $b_{1}$ und $b_{3}$ folgen aus den $y$ und $k$, $a_{1}$ und $a_{2}$ aus den Größen $T_{\odot}=6.25, m_{\odot}=-26.79$ (Potsdam), $m_{\odot}=-26.55$ (Harvard), und schließlich ergeben sich für $\alpha$ aus $x_{1}$ und $x_{2}$ zwei Werte, die sich gegenseitig kontrollieren. Die Rechnung führt $z u$ folgenden Gleichungen:

$$
\begin{aligned}
m_{P} & =-42.54+27.089 T^{-1}-5 \log \sin \varrho \\
m_{H} & =-42.97+28.237 T^{-1}-5 \log \sin \varrho \\
\text { I.3 } 3 m_{D} & =-42.39+36.393 T^{-1}-5 \log \sin \varrho
\end{aligned}
$$

wo $\alpha$ gleich dem arithmetischen Mittel der Werte -42.46 und -42.32 angenommen wurde. Die Vergleichung von $b_{1}$ und $b_{2}$ mit dem in A. N. 44 I 9 Gleichung (1 5 ) mitgeteilten Wert $b=28.6 \mathrm{I}$, der nur auf fünf Bestimmungen der Flächenhelligkeit des schwarzen Körpers beruht, führt zu dem wichtigen Resultat, daß die Sterngrößen $m_{P}$ und $m_{H}$ durch die Strahlung bei einer bestimmten Wellenlänge des schwarzen Körpers genügend dargestellt werden, dessen absolute Temperatur gleich ist der von Scheiner und Wilsing gefundenen effektiven Oberflächentemperatur $T_{2}$ der betreffenden Sterne.
Die Abweichung von $b_{1}$ respektive $b_{2}$ vom theoretischen Werte $b$ ist kleiner als die Differenz $b_{2}-b_{1}$, die durch die bekannte, von der Sternfarbe, folglich der Sterntemperatur abhängige systematische Differenz $m_{P}-m_{H}$ verursacht ist. Die für beide Kataloge charakteristischen Wellenlängen folgen aus (4), wenn statt $\beta \quad b_{1}$ respektive $b_{2}$ gesetzt wird; es wird dann für den Potsdamer Katalog $\lambda_{0}=0.585 \mu$, für Harvard $\lambda_{0}=0.56 \mathrm{I} \mu$. Gleichzeitig ist durch diese Rechnungen auf indirektem Wege die Gültigkeit des Wienschen Strahlungsgesetzes für die Draper-Größen mit ziemlicher Genauigkeit bewiesen. - Weitere Messungen der Flächenhelligkeit des schwarzen Körpers in einem möglichst ausgedehnten Temperaturgebiet und eine darauf gegründete Neuberechnung der Konstante $b$ wären sehr erwünscht.

Aus den Gleichungen (16) bis (18) lassen sich bei Kenntnis der entsprechenden Sterngrößen und der Temperatur die scheinbaren Radien $\varrho$ leicht berechnen. Ist $T$ unbekannt, so müssen zwei der dort eintretenden Kataloggrößen, am besten $m_{D}$ und $m_{P}$ oder $m_{D}$ und $m_{H}$ gegeben sein; dann folgt durch Elimination von $T$, wenn $\varrho$ in Sekunden ausgedrückt wird :

$$
\text { und } \begin{aligned}
\log \varrho^{\prime \prime} & =-3.280+0.7406 m_{D}-0.764 \mathrm{I}_{P} \dot{P} \\
\log \varrho^{\prime \prime} & =-3.668+0.8768 m_{D}-0.8679 m_{H} .
\end{aligned}
$$

Die Flächenhelligkeiten für die Sonne als Einheit: $h: h_{\odot}$ folgen auch leicht aus $(\mathrm{r})$, wenn $B_{1}$ und $B_{2}$ aus $b_{1}$ und $b_{2}$ berechnet werden, durch $\log \frac{h}{h_{\odot}}=B\left(\frac{\mathbf{I}}{T_{\odot}}-\frac{\mathrm{I}}{T}\right)$; nach Einsetzung der Konstanten wird:

$$
\log \frac{h}{h_{\odot}}=++_{1.734}-{ }_{\text {I } 0.8}{ }_{36} T^{-1}
$$

für Potsdamer Größen,

$$
\log \frac{h}{h_{\odot}}=+{ }_{1.807}-\mathrm{II.295} T^{-1}
$$

für Harvard-Größen. Zur Orientierung sollen die Werte in der folgenden Tabelle dienen, wo die $\varrho_{0}{ }^{\prime \prime}$ der nullten Größe entsprechen.

Tabelle III.

\begin{tabular}{r||l|l||l|l}
\hline \multicolumn{1}{c||}{} & \multicolumn{2}{c||}{ Potsdam } & \multicolumn{2}{c}{ Harvard } \\
\cline { 2 - 5 } & $h: h_{\odot}$ & \multicolumn{1}{c|}{$\varrho_{0}{ }^{\prime \prime}$} & $h: h_{\odot}$ & \multicolumn{1}{|c|}{$\varrho_{0}{ }^{\prime \prime}$} \\
\hline 3.0 & 0.0133 & $0.04 \mathrm{I}$ & $0.01 \mathrm{I} \mathrm{O}$ & 0.040 \\
4.5 & $0.2 \mathrm{I} 2$ & 0.010 & 0.198 & 0.0094 \\
6.0 & 0.847 & $0.005 \mathrm{I}$ & $0.84 \mathrm{I}$ & 0.0046 \\
9.0 & $3.3^{\prime \prime}$ & 0.0026 & 3.57 & 0.0022 \\
12.0 & 6.77 & 0.0018 & 7.35 & 0.0016
\end{tabular}

Hier ist die sehr rasche Zunahme von $h$ mit $T$ bei den niedrigeren Temperaturen sehr auffallend. Die großen Unterschiede der Flächenhelligkeit bedingen auch die große Verschiedenheit des scheinbaren Radius für dieselbe Größe. Für die Größenklasse $m$ läßt sich das entsprechende $\varrho$, wie leicht ersichtlich, durch Multiplikation der Werte $\varrho_{0}{ }^{\prime \prime}$ in der Tabelle mit Io $^{-1 / 5 m}$ berechnen. 
Interessant sind die so berechneten $\varrho^{\prime \prime}$-Werte für Sterne mit bekannter Parallaxe $p$, da in solchen Fällen auch der lineare Radius durch $\varrho^{\prime \prime}: p$ bestimmt ist, in der mittleren Entfernung von Erde-Sonne als Einheit. Wird diese Zahl mit $\operatorname{cosec} \varrho \odot=2$ I 4.9 multipliziert, so erhalten wir den Radius in Einheiten des Sonnenradius $r: r \odot$. Ohne eine systematische Übersicht des hierzu verwendbaren Materials geben zu wollen, habe ich für einige solche Sterne, die größtenteils absolut heller als die Sonne sind, die Rechnungen ausgefiihrt. Die Sterne sind meistens der Tabelle 6 a von Hertasprungs Abhandlung: Zur Strahlung der Sterne II $\left.{ }^{1}\right)$ entnommen, nur für die beiden ersten stammen die Parallaxen aus Kapteyns Zusammenstellung ${ }^{2}$ ). Die Größenangaben in der 3 . Spalte sind Harvard-Größen, dann folgen die $m_{D}-m_{H}$ und die Temperatur aus Tabelle I; wenn $T$ nicht bekannt ist, habe ich diese Größe nach (14) berechnet und diesen, nur als beiläufige Näherung zu betrachtenden Wert, in Klammern gesetzt. Die Parallaxen sind nach Kapteyns Vorgang unn o."008 vergrößert, um sie in absolute $z$ u verwandeln. Die nach $(\mathbf{2 0})$ berechneten $\varrho^{\prime \prime}$ stehen in der 7 . Spalte, die aus (17) folgenden in der 8. Spalte. $r: r \odot$ sind für den I., 2. und 6. Stern aus ( 17 ), für alle übrigen aus (20) berechnet. Die letzte Spalte enthält die absoluten Größen $M$ für $p=\mathrm{I}^{\prime \prime}$. Die absolute Größe der Sonne ist $-0^{\mathrm{m}} \mathbf{2 z}$.

Tabelle IV.

\begin{tabular}{|c|c|c|c|c|c|c|c|c|c|}
\hline Stern & Spektr. & $m_{H}$ & $m_{D}-m_{H}$ & $T$ & $p$ & $\varrho^{\prime \prime}$ aus $(20)$ & $\varrho^{\prime \prime}$ aus ( I 7$)$ & $r: r \odot$ & $M$ \\
\hline$\alpha$ Boot & $\mathrm{K}$ & 0.24 & - & $3 \cdot 5$ & ०."028 & - & o."c & I $5^{\circ}$ & 7 \\
\hline$\alpha$ Leonis & B 8 & 1.3 & - - & 9.2 & 0.0 & - & OOI I & 8.7 & $-6 i$ \\
\hline$\alpha$ Tauri & $\mathrm{K}_{5}$ & I.06 & +2.88 & $(2.4)$ & o. 17 & 0.07 .3 & - & ᄃ 35 & --3. \\
\hline$\gamma$ Cygni & F $8 p$ & $2 \cdot 3^{2}$ & +0.84 & $(6.8)$ & O. I 10 & 0.0012 & - & 2.4 & --2. \\
\hline$\gamma$ Ursae maj. & A & 2.54 & +0.35 & $(\mathbf{1} 2.5)$ & 0.103 & 0.00046 & $\cdots \cdot \cdot$ & 0.96 & -2. \\
\hline$\alpha$ Aquilae & A 5 & 0.89 & - & 7.1 & o & - & 0.0022 & I.9 & --2.2 \\
\hline$\beta$ Cassiopeiae & $F_{5}$ & 2.42 & $+1.2 \mathrm{I}$ & $(4.8)$ & o. $3^{8}$ & 0.0026 & - & 4.0 & $-\mathrm{r} .8$ \\
\hline$\chi \mathrm{D}$ & F 8 & 3.69 & +0.55 & $(6.3)$ & O. I I 3 & 0.00070 & - & I. 3 & $--\mathrm{r} . \mathrm{c}$ \\
\hline $5 . \mathrm{H}$ & G & 3.00 & +0.93 & $5 \cdot$ & 0.160 & 0.00 I 5 & 0.0014 & 2.0 & -0.9 \\
\hline$\mu \mathrm{H}$ & $G_{5}$ & $3 \cdot 4^{8}$ & +0.91 & 5.2 & 0.130 & 0.0015 & 0.0013 & 2.4 & --0.0 \\
\hline$\beta$ Virginis & F 8 & 3.80 & +0.60 & $(5.9)$ & $0.1 \mathrm{I} 8$ & 0.00078 & - & I. 4 & $-0 . \xi$ \\
\hline - Persei & $\mathrm{G}$ & $4 \cdot 17$ & $+0.7 x$ & $(5.0)$ & O.III & 0.00098 & - & I. 9 & -0.6 \\
\hline z Cygni & $\mathrm{F}$ & 3.82 & +0.49 & 7 & & 0.00062 & 0.0005 & 0.8 & -0.5 \\
\hline na & G & $4 \cdot 3$ & +0.84 & $(4 \cdot 4)$ & O. I I I & 0.00 I 3 & -- & 2.5 & -0.4 \\
\hline y Herculis & $\mathrm{K}$ & $3.6 \mathrm{I}$ & +1.04 & 5.1 & O. I 58 & 0.0019 & 0.0013 & 2.6 & -0.1 \\
\hline$\iota$ Piscium & $\mathrm{F}_{5}$ & 4.28 & +0.72 & $(4.8)$ & 0.146 & 0.0010 & - & 1.5 & +0.1 \\
\hline$\hat{\lambda}$ Aurigae & $\mathrm{G}$ & 4.85 & +0.39 & $(5.6)$ & O.II 7 & 0.00052 & - & 0.96 & +0.1 \\
\hline
\end{tabular}

Es folgt aus diesen Beispielen, daß die wahren Halbmesser der Sterne meistens von gleicher Größenordnung sind, wie der Sonnenradius, nur bei den hellsten Sternen erreichen sie wesentlich größere Beträge, wenn die Parallaxe sehr klein oder die Temperatur sehr niedrig ist, besonders wenn beide Ursachen zusammenwirken.

Vor Anwendung der obigen Formeln wäre hier eigentlich noch der etwaige Einfluß des Faktors $i-e^{-\frac{c_{2}}{\lambda T}}$ zu diskutieren, durch den die Reduktion der Wienschen Formel auf die Plancksche dargestellt wird. Der Einfluß dieses Faktors macht sich bei der genauen Berechnung der Energie im Spektrum bei höheren Temperaturen jedenfalls geltend und wurde auch von Scheiner und Wilsing überall, wo nötig, berücksichtigt. In den Gleichungen von der Form $(7)$ and $(r)$ fällt jedoch dieses Korrektionsglied aus den Differenzen der Sterngrößen fast vollständig heraus und erreicht selbst bei den höchsten Sterntemperaturen nur wenige hundertstel Größenklassen, was bei der hier erstrebten Genauigkeit kaum in Frage kommt. Bei den Gleichungen ( 16 ) bis (18) wäre dieser Einfluß schon wesentlich größer, ich glaube aber auch hier dieses Glied füglich vernachlässigen zu dürfen, da die mäßige Genauigkeit der benutzten Konstanten eine entsprechende Genauigkeit der Rechnung kaum gestattet. Zur Orientierung möchte ich nur erwähnen, daß die Einführung dieses Gliedes in $(2 x)$ und $(22)$ die beiden letzten Werte von $h: h_{\odot}$ in der Tabelle III um rund 7 respektiveI 4 Prozent erhöhen würde.

Als Anwendung der mitgeteilten Formeln soll die Abhängigkeit der im Potsdamer Katalog gegebenen Sternfarben von der Temperatur ermittelt werden. $\mathrm{Zu}$ diesem $\mathrm{Zwecke}$ habe ich die 95 Sterne der Tabelle I nach den Hauptfarben gruppiert und das Mittel der $m_{D}-m_{P}$ für jede Gruppe gebildet, daraus die mittleren Temperaturen nach (Io) berechnet. Diese Werte nebst der Anzahl der benutzten Sterne stehen in den Spalten $\mathrm{I}$ bis 4 der folgenden Tabelle. Um $m_{P}-m_{H}$ darzustellen, kann die Differenz von ( 16 ) und $(\mathrm{r} \eta)$ :

$$
m_{D}-m_{H}=+0^{\mathrm{m}} 43-\mathrm{T} .148 T^{-1}
$$

dienen. Die daraus folgenden Werte stehen in der 6. Spalte, in der 5: sind die von Müller und Kempf nach der Formel ${ }^{3}$ ),

1) Zeitschrift für wissenschaftl. Photographie Bd. V p. 87 (1907).

3) On the mean parallax of stars of determined proper motion and magnitude. Publications of the astron. Laboratory at Groningen. No. 8 p. 26-27.

3) Publikationen des Astrophys. Observatoriums zu Potsdam Bd. I 7 p. XXXIV. 


$$
P-H=+0^{\mathrm{m} 229}+0^{\mathrm{m}} \mathrm{2}_{4}\left(\text { Größe }-2^{\mathrm{m}} \mathrm{2}_{5}\right)-0^{\mathrm{m}} .027 \text { Farbe }-0^{\mathrm{m}} .008 \text { (Größe - } 2^{\mathrm{m}} \mathbf{2}_{5} \text { ) Farbe }
$$

für die Größen $3 \cdot 50-3^{\mathrm{m}} \cdot 99$ berechneten Werte eingetragen, die auf sehr reichhaltigem, doch etwas verschiedenem Material beruhen.

\begin{tabular}{|c|c|c|c|c|c|c|}
\hline \multirow{2}{*}{$\begin{array}{l}\text { An- } \\
\text { zahl }\end{array}$} & \multirow{2}{*}{ Farbe } & \multirow{2}{*}{$m_{D}-m_{P}$} & \multirow{2}{*}{$T$} & \multicolumn{3}{|c|}{$m_{P}-m_{H}$} \\
\hline & & & & $\operatorname{nach}(24)$ & $\operatorname{nach}(23)$ & nach $(25)$ \\
\hline 9 & W & +0.098 & 8.64 & $+0^{\mathrm{m}} 27$ & $+0^{\mathrm{m}} \cdot 297$ & $+0^{m} \cdot 266$ \\
\hline 36 & GW & $+0.25^{8}$ & $7 \cdot 3 \mathrm{I}$ & +0.23 & +0.273 & +0.237 \\
\hline 25 & WG & +1.024 & $4.2 \mathrm{I}$ & $+0 . \mathrm{II}$ & +0.157 & +0.099 \\
\hline 25 & G & +1.374 & 3.53 & +0.03 & +0.105 & +0.037 \\
\hline
\end{tabular}

Die Übereinstimmung von (2.3) mit den gegebenen Werten ist eine mäßige, doch mit Rücksicht auf die Unsicherheit der Koeffizienten genügende; wird die erste Konstante in $\left(2_{3}\right)$ auf +0.38 verkleinert, so wird die Darstellung ganz befriedigend. Noch genauer ist folgende Formel:

$$
m_{P}-m_{H}=+0.4^{2} 5-1.37 \text { I } T^{-1}
$$

deren Koeffizienten nach der Methode der kleinsten Quadrate durch Anschluß an die Werte von Müller und Kempf berechnet worden sind. Die daraus folgenden Werte in der letzten Kolumne stimmen mit den Zahlen der 5. Kolumne fast vollkommen überein. Es können somit die von der Sternfarbe abhängigen Differenzen der beiden untersuchten Kataloge als Funktion der Temperatur genügend dargestellt werden. Diese Untersuchung konnte wegen ungenügenden Materiales nicht auf die kleineren Potsdamer Farbenstufen W, $W+, G W-$ etc. ausgedehnt werden.

Zum Schlusse wäre hier noch die Frage zu erörtern, wie weit die obigen Resultate durch die etwaige Absorption des Lichtes im Weltraume beeinflußt werden. Trotz des negativen Resultates meiner diesbezüglichen Rechnungen möchte ich eine Methode an der Hand der beigegebenen schematischen Figur kurz erläutern, die meiner Ansicht nach bei einem größeren Material am einfachsten zum Ziele führt.

Wenn nach Scheiner und Wilsing $\left.{ }^{1}\right) E_{\lambda}^{\prime}$ die Intensität der bei den spektralphotometrischen Messungen gebrauchten Lampe für die Wellenlänge $\lambda$ bedeutet, $E_{\lambda}$ die entsprechende Intensität für den Stern und $n_{\lambda}$ das Verhältnis $E_{\lambda}: E_{\lambda}^{\prime}$ ist, und der Kürze halber

$$
\begin{aligned}
& \text { nd der Kürze halber } \\
& \log I_{\lambda}=\log n_{\lambda}+5 \log \lambda+\log E_{\lambda}^{\prime}+\log \left(x-e^{-\frac{c_{2}}{\lambda T}}\right)
\end{aligned}
$$

gesetzt wird, so ist die Temperatur $T$ des Sternes aus der transzendenten Gleichung:

$$
\log I_{\lambda}=x-\frac{c_{2} \log e}{\lambda} T^{-1}
$$

bestimmt, wo das vierte Glied in $\log T_{\lambda}$ mit einem Näherungswert ermittelt wird, wodurch dann $(26)$ in $T^{-1}$ linear wird. Werden jetzt die $\log I_{\lambda}$ als Ordinaten zu den $\frac{\mathrm{I}}{\lambda}$ proportionalen Abszissen aufgetragen, so stellt die Gleichung (26) eine Ge- rade dar, deren Richtungstangente proportional $T^{-1}$ ist. Es sei diese Gerade im beobachteten Spektralgebiet durch $A A^{\prime}$ dargestellt; falls die Absorption unmerklich wäre, dürften die $\log I_{\lambda}$ nur um Beträge abweichen, die den zufälligen Beobachtungsfehlern entsprechen. Wäre jedoch die Absorption merklich, so würden die wahren Beträge von $I_{\lambda}$ im Verhältnis $p_{\lambda}^{\Delta}$ verkleinert werden, wo $p_{\lambda}$ den Absorptionskoeffizienten und $\alpha$ die Entfernung des Sternes bedeuten. Es müßten somit die Ordinaten von $A A^{\prime}$ um die entsprechenden Beträge $-A \cdot \log p_{\lambda}$ vermindert werden, und die so erhaltenen Punkte kämen auf die Kurve $B B^{\prime}$ zu liegen, deren Gestalt zwar nicht genau bekannt ist, weil über $\not \lambda$ gegenwärtig nur Hypothesen aufgestellt werden können, doch müßte nach Kapteyns Annahmen $B B^{\prime}$ die konvexe Seite $A A^{\prime}$ zukehren, wenn $-\log p_{\lambda}$ schneller als $\frac{K}{\lambda}$ zunimmt, wo $K$ konstant ist.

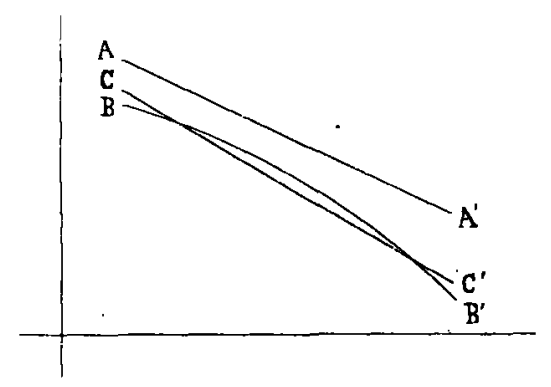

Werden die längs $B B^{\prime}$ liegenden Punkte nach (26) ausgeglichen, so wird die so ermittelte Gerade $C C^{\prime}$ im allgemeinen stärker gegen die Abszissenachse geneigt sein als $A A^{\prime}$ und somit einer umso niedrigeren Temperatur entsprechen, je größer ceteris paribus $\mathcal{A}$ ist. Bei einer Kurve von ähnlicher Gestalt müßten die Abweichungen im Sinne Beobachtung - Rechnung einen deutlich systematischen Gang zeigen, der sich mit $A$ vergrößert, indem die Abweichungen am roten Ende des Spektrums negativ wären, in der Mitte vorwiegend positiv und im Violett wieder negativ. Um diese Frage zu untersuchen, sind in der letzten Spalte der Tabelle I die totalen Eigenbewegungen $s$ nach Kapteyn ${ }^{2}$ ) eingetragen. Danach habe ich die Sterne in vier Gruppen geteilt, die Mittel der $s$ berechnet, daraus die mittleren Parallaxen nach Kapteyns Formel ${ }^{3}$ ):

$$
\bar{p}_{s m}=(0.905)^{m-5.5}[0.0387 s]^{\frac{1}{1.405}}
$$

für $m=3.5$ ermittelt und schließlich die mittleren Entfernungen $A$ in Kapteyns Einheit, $d=$ I für $p=\mathrm{O}^{\prime \prime} \mathrm{I}$, berechnet. Diese Größen stehen in den sechs ersten Spalten der Tabelle VI. Dann folgen die Mittel der entsprechenden Abweichungen $v_{2}$ nach Scheiner und Wilsing ${ }^{4}$ ) für die fünf beobachteten Wellenlängen in Einheiten der dritten Stelle von $\log I_{\lambda}$.

1) Publikationen des Astrophys. Observatoriums zu Potsdam Nr. 56 p. 42.

2) Publications of the astron. Laboratory at Groningen No. 9 .

3 ) Publications of the astron. Laboratory at Groningen No. 8 p. $28 . \quad$ t) Ebenda p. 48-62. 
Tabelle VI.

\begin{tabular}{|c|c|c|c|c|c|c|c|c|c|c|}
\hline \multirow{2}{*}{ Gruppe } & \multirow{2}{*}{$\begin{array}{l}\text { An- } \\
\text { zahl }\end{array}$} & \multicolumn{2}{|c|}{ Eigenbewegung } & \multirow{2}{*}{$p$} & \multirow{2}{*}{$\Delta$} & \multirow{2}{*}{$0.448 \mu$} & \multirow{2}{*}{$0.480 \mu$} & \multirow{2}{*}{$0.513 \mu$} & \multirow{2}{*}{$0.584 !$} & \multirow{2}{*}{$0.63^{8} \vdash$} \\
\hline & & Grenzen & Mittel & & & & & & & \\
\hline I & 23 & $0.00-0.050$ & 0.0282 & o.00952 & 10.5 & $-7 \cdot 3$ & +6.8 & -2.9 & $+\mathrm{I} .0$ & -4.4 \\
\hline II & 23 & $0.051-0.100$ & $0.079^{\circ}$ & 0.0198 & 5.05 & $-3 \cdot I$ & +3.2 & +0.7 & +0.7 & $-3 \cdot 7$ \\
\hline IIII & I9 & $0.101-0.200$ & $0.15 \mathrm{I}$ & 0.0314 & 3.18 & -2.8 & -4.7 & +8.7 & -0.2 & -3.2 \\
\hline IV & $3^{6}$ & $0.20 \mathrm{I}<s$ & $0.48 \mathrm{I}$ & $0.07 \times 7$ & I. 40 & -0.3 & $-\mathrm{I} .8$ & -1.2 & +7.1 & -6.7 \\
\hline
\end{tabular}

Bei der Beurteilung dieser Tabelle muß berücksichtigt werden; daß der mittlere Fehler eines Energie-Logarithmen \pm 33 beträgt und somit den hier stehenden Mittelwerten, je nach der Anzahl der Sterne, ein mittlerer Fehler von \pm 5 bis \pm 7 Einheiten anhaftet. In diesen Zahlen können noch etwaige systematische Fehlerreste enthalten sein, die nur von $\lambda$ abhängen und von der Abweichung des Vergleichsspektrums vom Planckschen Gesetze herrühren. Der größte Teil dieser Fehler ist durch die von Scheiner und Wilsing empirisch ermittelten Korrektionen weggeschafft worden. Bei der kleinen Entfernung der Gruppen III und IV sollten die entsprechenden Differenzen merklich ubereinstimmen und fast nur diese systematischen Fehler darstellen, bei den übrigen Gruppen für

jede Wellenlänge einen deutlichen Gang mit $d$ zeigen. Dieser Gang ist nur bei $0.44^{8} \mu$ angedeutet, sonst nicht vorhanden, und auch in den Horizontalreihen schwanken die Zahlen unregelmäßig auf und $a b$, ohne den Gang zu zeigen, der nach den obigen Betrachtungen zu erwarten wäre. Es läßt sich somit aus diesem Material nichts Bestimmtes über die Absorption folgern. Es wäre noch zu bemerken, daß die Abweichungen $v_{2}$ wesentlich kleiner sein müssen als der Gesamtbetrag der Absorption für das betreffende $\lambda$ und nur Einflüsse differentieller Art darstellen, die bis auf die zufälligen Beobachtungsfehler verschwinden würden, wenn $\log p$. gerade proportional $z \mathfrak{u} \frac{\mathrm{I}}{\lambda}$ variieren würde.

Budapest, 19 ro Juli 2.

B. v. Harkányi.

\section{Veränderliche Sterne im Orionnebel.}

Im A. I. 26. I 38 berichtet Herr $K$. Burns in Minneapolis, daß acht photographische Aufnahmen des Orionnebels die Veränderlichkeit von einigen früher in den Harvard-Zirkularen 78 und 86 (A. N. I65.2 I 5 und I66.3 I5) angezeigten Variabeln bestätigen, nämlich von $53,7 \mathrm{x}, 73,74,95,97$, 102 und I 8.1904 Orionis, ferner, daß die folgenden Sterne veränderlich oder, soweit mit * versehen, der Veränderlichkeit verdächtig seien.

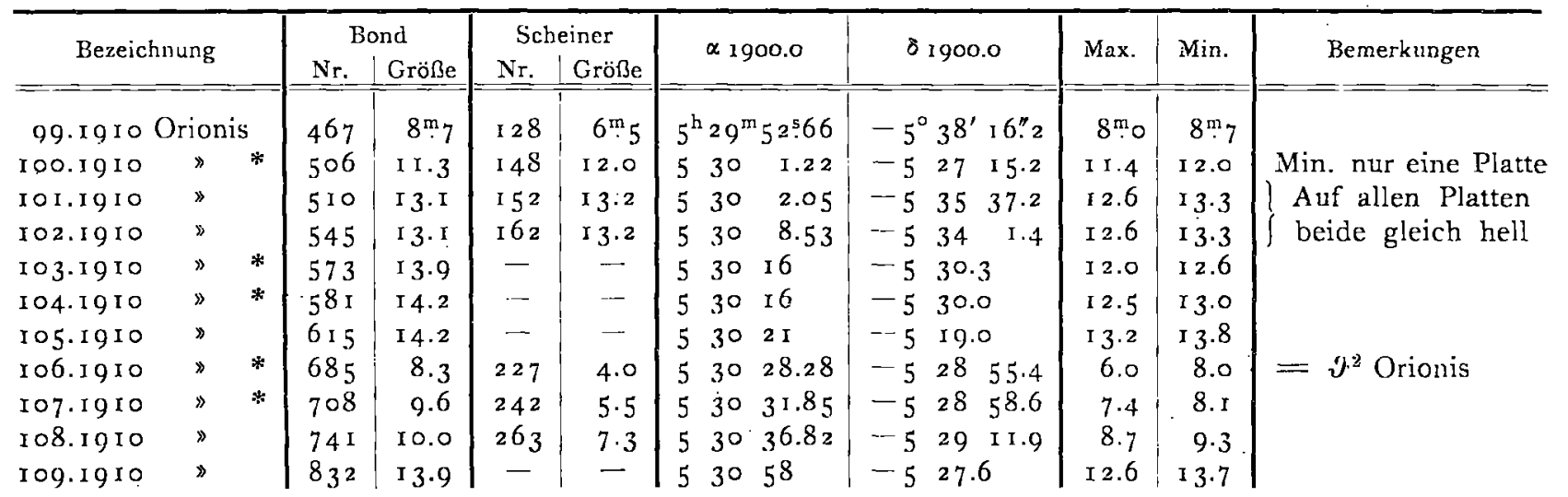

Die Angaben unter »Bond " beziehen sich auf G. P. Bonds Vermessung des Großen Orionnebels (Harv. Ann. 5) und die unter "Scheiner" auf die Arbeit in Potsd. Publ. I I. Aus letzterer Publikation sind auch die genauen Örter entnommen.

Die Anzahl der Variabeln im Orionnebel, die nach Kreutz (A. N. 166.317) im Jahre 1904 133 betrug, ist seitdem durch Hinzukommen von $2-7,36-39$, I $24-$ r 25.1906 Orionis und der obigen auf 156 gestiegen.

Eb.

(471) Papagena. Herr G. Strömberg, Assistent an der Sternwarte zu Stockholm, bittet Beobachtungen des. Planeten (471) Papagena aus dem Jahre I910 ihm einzusenden zur definitiven Bahnbestimmung des Planeten.

Inhalt zu Nr. 445r. B. v. Harkányi. Darstellung der photometrischen und photographischen Größe als Funktion der Temperatur der Sterne. 16I. - Veränderliche Sterne im Orionnebel. 175. - (47I) Papagena. 175. 